\title{
Recuperación de las muestras en estudios longitudinales con adolescentes a través de las redes sociales: el estudio ILSEG
}

\author{
Rosa Aparicio ${ }^{1}$; Andrés Tornos $\dagger^{2}$
}

Recibido: 29 de febrero de 2020 / Aceptado: 19 de junio de 2020

Resumen. Los estudios longitudinales de "panel" en los que se trata de seguir los cambios que se producen en el tiempo en una determinada población siguiendo a una misma muestra de esa población en distintos momentos de su vida, tropiezan con una especial dificultad: la de ir perdiendo en cada nueva fase de seguimiento un gran número de los sujetos que componían la muestra inicial.

El artículo que sigue examina esta situación en relación con los estudios longitudinales dirigidos a adolescentes y jóvenes mostrando el modo original de enfrentarse con ella que adoptó el estudio ILSEG (Investigación Longitudinal sobre la Segunda Generación) recurriendo a las posibilidades ofrecidas por las redes sociales Facebook y Tuenti. Trata pues los aspectos teóricos, metodológicos y prácticos de las posibilidades que ofrece internet para localizar y contactar a informantes conocidos. De hecho, sin el auxilio de las redes sociales de Internet, ILSEG no habría podido localizar sino al 44\% de su muestra inicial de hijos de inmigrantes. Tras el recurso a internet se pudo llegar a localizar y a encuestar a un $73 \%$ : una cifra ampliamente satisfactoria en esta clase de estudios.

Palabras clave: Hijos de inmigrantes; estudios longitudinales con adolescentes; metodología de investigación; seguimiento de muestras; redes sociales en internet.

\section{[pt] Recuperação de amostras em estudios longitudinais com adolescentes através de redes sociais: o estudo ILSEG}

Resumo. Os estudos longitudinais do "painel", no qual ele tenta acompanhar as mudanças que ocorrem ao longo do tempo em uma dada população, seguindo a mesma amostra dessa população em diferentes momentos de sua vida, enfrentam uma dificuldade especial: a de ir perdendo em cada nova fase do acompanhamento um grande número de sujeitos que compuseram a amostra inicial.

O artigo a seguir examina essa situação em relação aos estudos longitudinais voltados para adolescentes e jovens, mostrando a maneira original de lidar com ela que adotou o estudo ILSEG (Pesquisa Longitudinal de Segunda Geração), utilizando as possibilidades oferecidas pelas redes sociais Facebook e Tuenti Ele trata dos aspectos teóricos, metodológicos e práticos das possibilidades oferecidas pela internet para localizar e entrar em contato com informantes conhecidos. De fato, sem a ajuda de redes sociais na Internet, o ILSEG não poderia ter localizado mais de $44 \%$ de sua amostra inicial de filhos de imigrantes. Após o uso da internet, 73\% puderam ser localizados e pesquisados: um número amplamente satisfatório nesse tipo de estudo.

Palavras chave: Filhos de imigrantes; estudos longitudinais com adolescentes; metodologia de pesquisa; rastreamento de amostras; redes sociais na internet.

\section{[en] Sample Recovery in longitudinal Studies with Adolescents using Social Networks: the ILSEG Study}

Abstract. Panel studies which consist in following the changes produced over time in a specific population by following the same sample of this population at various stages in their life, stumble with a special difficulty: that is, the risk of losing a large number of the subjects which were part of the initial sample in each of the follow-up stages.

The paper which follows examines this situation in relation to longitudinal studies with adolescents and young people. It exposes the original way adopted by ILSEG (spanish acronym of the Longitudinal Study of the Second Generation) to

\footnotetext{
Instituto Universitario José Ortega y Gasset (rag.migraciones@fogm.es).

E-mail: raparicio@comillas.edu

Universidad Pontificia Comillas. 
face this problem, resorting to the possibilities offered by social networks such as Facebook and Tuenti. The chapter thus considers the theoretical, methodological and practical possibilities offered by internet for tracking and contacting known informants. In fact, without the help of the social networks in internet, ILSEG would only have been able to recuperate $44 \%$ of its initial sample of children of immigrants. After recurring to internet, it was able to trace and to interview $73 \%$ of the sample, an amply satisfactory number in this kind of studies.

Keywords: Children of immigrants; longitudinal studies with adolescents; research methodology; sample tracing; social networks in internet.

Sumario. 1. Introducción. 2. La atrición de la muestra en los estudios longitudinales con niños y adolescentes y el rastreo de los individuos de la muestra en las fases de seguimiento: estado de la cuestión. 3. El estudio de la integración de los hijos de inmigrantes y la Investigación Longitudinal de la Segunda Generación (ILSEG). 3.1. La encuesta inicial. 3.2. La primera encuesta de seguimiento y los problemas de recuperación de la muestra. 4. El papel de las redes sociales por internet en la recuperación de la muestra origina. 5. Conclusiones. 6. Referencias bibliográficas.

Cómo citar: Aparicio, R.; Tornos, A. (2020): Recuperación de las muestras en estudios longitudinales con adolescentes a través de las redes sociales: el estudio ILSEG, Sociedad e Infancias, 4, 23-34.

\section{Introducción}

Uno de los mayores riesgos, sino el mayor ${ }^{3}$, en los estudios longitudinales en los que se pretende seguir a unos mismos individuos durante un periodo de tiempo prolongado es el de que se produzca una importante pérdida de casos en las sucesivas recolecciones de información. Ese riesgo existe aun cuando los investigadores, adelantándose a esas posibles pérdidas, hayan tomado todas las medidas posibles para minimizarlo en los diferentes pasos de diseño de su investigación. Y este riesgo es aún mayor cuando las poblaciones objeto del estudio están expuestas a múltiples cambios como ocurre con los niños, con los adolescentes o con los inmigrantes. Es así como frecuentemente ocurre, y más en estos casos, que no dan suficiente resultado los medios que se habían previsto para localizar en las fases de seguimiento a los individuos de la muestra inicial. Ello obliga entonces a los investigadores a recurrir a estrategias para rastrearlos no contempladas previamente, bien inspirándose en el know how existente en esta materia a través de la literatura sobre el tema o bien ideando algo nuevo.

El artículo que sigue examina esta situación en relación con los estudios longitudinales de panel dirigidos a adolescentes mostrando el modo original de enfrentarse con ella que adoptó el estudio ILSEG (Investigación Longitudinal sobre la Segunda Generación) $)^{4}$ recurriendo a las posibilidades ofrecidas por las redes sociales Facebook y Tuenti. Trata pues los aspectos teóricos, metodológicos y prácticos de las posibilidades que ofrece internet para localizar y contactar a informantes conocidos.

\section{La atrición de la muestra en los estudios longitudinales con niños y adolescentes y el rastreo de los individuos de la muestra en las fases de seguimiento: estado de la cuestión}

Si queremos obtener información acerca de lo que le ha ocurrido sucesivamente en el tiempo a los individuos de una determinada población podemos recurrir a un estudio transversal en el que, además de preguntarles sobre acontecimientos en el momento en que se realiza el estudio, se les pregunta acerca de los acontecimientos del pasado sobre los que deseamos obtener información. Y esto es lo que con más frecuencia se hace, en buena parte debido al alto coste que puede suponer el realizar estudios que sigan a los individuos en diversos momentos de su evolución en el tiempo. Pero la información así obtenida sobre situaciones del pasado corre el peligro de no ser del todo fiel a esas situaciones bien porque falla la memoria o porque lo que se recuerda se ve distorsionado por la situación presente. Para evitar dicha limitación la posibilidad es recurrir a estudios longitudinales siempre que esto sea posible, entendiendo por estudio longitudinal una investigación en la que se llevan a cabo mediciones continuas o repetidas de un fenómeno determinado durante periodos de tiempo prolongados. ${ }^{5}$ Los estudios longitudinales ofrecerían además las ventajas, al poder seguir la evolución de los sujetos o del fenómeno en el tiempo, de permitir conocer los cambios habidos así como de poder establecer relaciones causales.

Ahora bien, es sabido que los estudios longitudinales pueden adoptar o bien la metodología de estudios de panel o bien la de estudios de cohorte. De cual se elija dependerá el tipo de información de la que podremos disponer. Así, en los estudios de cohorte, diferentes muestras de una misma población serían entrevistadas en periodos de tiempo sucesivos. En cambio, en los estudios de panel, una misma muestra es entrevistada a través del tiempo en momentos

\footnotetext{
"Attrition is the Achilles heel of longitudinal studies" (Thomas et al., 2012: 108).

Aparicio y Portes (2014); Portes y Aparicio (2016).

En esto coinciden todos los estudiosos del tema. Véase, por ejemplo, Menard (2007). Introduction: Longitudinal Research: Design and Analysis.

En S. Menard (Editor), Handbook of Longitudinal Research: Design, Measurement, and Analysis (pp. 3-12). Nueva York: Elsevier.
} 
clave de transición a lo largo de la vida. Este método permite seguir los cambios individuales a través del tiempo e identificar sus determinantes. El método de cohorte, por el contrario, no permite esto. Solo puede identificar los cambios grupales que se han producido con el tiempo y destacar los factores colectivos que han llevado a ellos. ${ }^{6}$

Sin embargo, los estudios de panel, precisamente porque suponen seguir a unos mismos individuos a través del tiempo, deben enfrentarse con las dificultades inherentes a tener que volver a contactar con dichos individuos en sucesivos momentos a lo largo de sus vidas. Y esta dificultad se vería acrecentada tratándose de poblaciones o de individuos que por su situación o naturaleza tienen mayor tendencia a la movilidad como puede ocurrir con los inmigrantes o con los que coinciden etapas en que las que estarán experimentando importantes cambios en sus vidas como ocurriría con los niños o adolescentes. ${ }^{7}$

Para la mayoría de los autores este sería el mayor riesgo al que estarían expuestos los estudios longitudinales con el método de panel y este riesgo sería aún mayor en los estudios con adolescentes. ${ }^{8} \mathrm{Y}$ ello porque las dificultades en el seguimiento pueden producir una excesiva mortandad de la muestra debido a los cambios de situación que ocurren en la adolescencia, la cual no solo redunde en una reducción importante de la muestra con la consiguiente pérdida de potencia estadística que ello puede entrañar, sino que también la pérdida puede ser selectica lo que daría lugar a serios sesgos en la muestra resultante, es decir, que esta no llegue a representar en todos sus aspectos a la muestra original $\mathrm{y} / \mathrm{o}$, selectivamente en unos aspectos determinados. Cuanto mayor sea el tiempo transcurrido entre la encuesta inicial y las encuestas sucesivas, más significativos serán sus resultados; pero también será mayor el riesgo de una importante pérdida de casos. Por ejemplo, un estudio de panel entre adolescentes en los dos primeros cursos de la ESO con un periodo de un año entre la muestra original y la de seguimiento probablemente recuperará una proporción importante de los originalmente encuestados, pero es improbable que proporcione resultados significativos ya que poco cambio ha podido producirse en tan corto espacio de tiempo. Por el contrario, un espacio de tres o más años puede poner al descubierto cambios importantes relacionados con el tiempo en esta joven población, aunque corre el riesgo de una importante disminución de la muestra. ${ }^{9}$

Es abundante la literatura sobre el tema. Esta, sin embargo, ha tendido a centrarse en cómo corregir los sesgos producidos por dicha pérdida antes que en tratar de ver cómo evitar la pérdida de casos de la muestra. No obstante, sí hay autores que se han preocupado de estudiar este tema, bastantes de ellos específicamente en relación con los estudios sobre adolescencia y niñez. Lo que estos autores en su mayoría hacen es analizar las diversas estrategias utilizadas en múltiples estudios longitudinales sobre adolescencia y niñez para evitar o minimizar la perdida de casos en las sucesivas oleadas llevadas a cabo por estas investigaciones tratando así de determinar cuáles han sido más o menos exitosas.

Lo que destaca de los análisis realizados por estos autores es que los investigadores despliegan numerosas estrategias para intentar reducir al máximo la pérdida de individuos de la muestra en las fases de seguimiento de los estudios longitudinales y que esas estrategias deben realizarse desde la fase de diseño, en la fase inicial y fases de seguimiento de la investigación, sin olvidar los tiempos entre medias. Un metaanálisis realizado por varios autores ${ }^{10}$ de 143 investigaciones longitudinales, clasifica las estrategias utilizadas en cuatro grandes categorías: estrategias encaminadas a la reducción de barreras, a la formación de comunidad, al recordatorio del seguimiento y al rastreo de casos. La primera categoría se referiría sobre todo al diseño y a la fase inicial de la investigación: a que el instrumento que se utilice para la obtención de información sea lo más asequible posible para la población a la que va dirigido, a la formación del personal para establecer una buena relación con los entrevistados y a aquellas acciones dirigidas a asegurar desde el principio la obtención de todos los datos necesarios como nombres, direcciones, teléfonos, correo electrónico, etc. para poder localizar a las personas que van a conformar la muestra en las futuras oleadas del estudio. La formación de comunidad aludiría a acciones orientadas a motivar desde el principio y a mantener motivados a los sujetos de la muestra y a su entorno llevándolos a identificarse con la investigación. La tercera de las categorías consideraría las actividades llevadas a cabo entre oleadas para recordar cuando se producirá la siguiente fase de seguimiento. Finalmente, la cuarta categoría abarcaría las estrategias utilizadas para rastrear a los individuos en cada una de las sucesivas fases de seguimiento.

Son las estrategias dentro de esta última categoría las que aquí nos interesan dado el foco de este artículo en el que, como se indicó en la introducción, lo que se pretende es exponer la novedad y utilidad de las redes sociales como estrategia de rastreo en el seguimiento de los adolescentes de una muestra, ilustrándolo con su uso en la Investigación Longitudinal sobre las Segundas Generaciones (ILSEG).

Tanto los autores del metaanálisis citado como otros autores apuntan al uso habitual por las investigaciones longitudinales examinadas de una combinación de estrategias de rastreo en el seguimiento de los individuos de una muestra. La coincidencia entre ellos será alta en las estrategias concretas nombradas. Lo más habitual es una secuencia de

\footnotetext{
Firebaugh, 2008.

"Sample attrition, a major concern in any longitudinal study, is even more problematic when adolescents are the study population" (Stephens et al, 2002). Y también: "The value of longitudinal studies has to be judged against the problems of collecting longitudinal data. The most serious of these data quality issues is "attrition", the loss of sample members over time" (Bynner, 1996).

$8 \quad$ Boys et al., 2003; Delgado y Llorca, 2004; Dworsky, 2014; Epstein y Botvin, G.J., 2000; Hobden et al., 2011; King y Churchill, 2000; Loeber y Farrington, 1994.

9 Firebaugh, 2008. Para una discusión de las contrapartidas en los diseños longitudinales de estudio de la Segunda Generación, ver Portes et al., 2001, cap. 2; y Haller et al., 2011.

10 Teague et al., 2018.
} 
acciones en las que, a grandes rasgos, a) primero se intenta localizar a los individuos en el teléfono o la dirección del domicilio o del correo electrónico que hubieran proporcionado; b) si ello no funciona, se intenta localizarlos a través de los autores que denominan contactos colaterales, es decir, a través de otros participantes o de entidades o personas del entorno que pudieran tener una relación con esos individuos; c) simultánea o seguidamente, en muchos casos se ha intentado actualizar las direcciones y teléfonos de los individuos no localizados a través de bases de datos de pago en internet o a través de servicios administrativos (como datos de permisos de conducir, número de la seguridad social, etc.). Unido a todo esto, para motivar a los individuos a participar, en bastantes de los casos, se establece algún tipo de incentivo que puede ser económico o de otra clase según la población de que se trate y los medios de los que se dispone para la investigación.

Es necesario señalar que la gran mayoría de las investigaciones a las que aluden los autores han sido realizadas en países anglófonos como Estados Unidos, Canadá, Australia o Gran Bretaña, países en los que se dispone de varios recursos administrativos y de bases de datos públicas para localizar a los sujetos, recursos que no están disponibles en España. De ahí que el paso reseñado en el epígrafe c) no sería aquí una alternativa. Por otra parte, en ningún caso de los analizados por los distintos autores aparecen nombradas las redes sociales como uno de los recursos utilizados por las investigaciones longitudinales y como recurso posible para el rastreo en el seguimiento de los individuos de una muestra en estudios longitudinales.

\section{El estudio de la integración de los hijos de inmigrantes y la Investigación Longitudinal de la Segunda Generación (ILSEG)}

El interés por la integración de la población inmigrada, en los países a que ésta había llegado en mayor número, se extendió muy pronto a una especial consideración de la integración paulatina de los hijos de éstos, al contarse, por una parte, con que esta generación de los hijos sería la que podría innovar establemente la composición demográfica y social del país -y por otra parte con que esta generación de los hijos, al no haberse socializado en el país de origen de sus padres, mostrarían más claramente el potencial integrador de las políticas e instituciones concebidas para su mejor inserción en los nuevos contextos.

Pero, los estudios que podrían ilustrarnos mejor sobre las trayectorias de inserción de los hijos de inmigrantes no serían los transversales, aunque ellos pueden darnos una información básica sobre momentos clave de dicha inserción. Sí lo serían en cambio los estudios longitudinales, y más en concreto los estudios de panel, ya que como se ha señalado, son los más adecuados para captar los frecuentes cambios que caracterizan el normal desarrollo de los adolescentes y jóvenes, así como para establecer las causas de esos cambios.

Ahora bien, los estudios longitudinales que suponen el seguimiento de una misma muestra a lo largo del tiempo pueden presentar, como se ha venido reiterando, serios problemas de mortandad $^{11}$ de la muestra que pueden llegar a invalidar los resultados de una investigación. La consecuencia es que toda investigación longitudinal ha de empeñarse en encontrar medios para reducir al máximo las posibles pérdidas de casos que se pueden producir en las sucesivas fases de seguimiento.

Una de las primeras investigaciones longitudinales sobre hijos de inmigrantes, si no la primera, fue el estudio CILS (Children of Immigrants Longitudinal Study) llevado a cabo en Estados Unidos por Alejandro Portes y Rubén Rumbaut, Este estudio modélico había seguido desde la primera adolescencia hasta la adultez temprana (de 1991 a 2001) a hijos de inmigrantes en las áreas metropolitanas de Miami (Florida) y de San Diego (California) con el fin de conocer sus procesos de adaptación. Por iniciativa de Portes, en el año 2007 se propuso replicar dicho estudio en España lo que dio lugar al estudio ILSEG (Investigación Longitudinal sobre la Segunda Generación) iniciada en ese año y terminada en 2017. ${ }^{12}$ En España, se trataba de seguir en sus etapas claves, desde la primera adolescencia hasta la adultez temprana, el progreso en la integración de los hijos de los inmigrantes extranjeros llegados a nuestro país.

El estudio consistía por tanto en un estudio de panel que debía tener tres fases. En la primera, se trataba de obtener información relativa a la integración de los hijos de inmigrantes que en ese momento tuvieran una media de edad entre los 13 y 14 años. En la segunda se pretendió seguir a esos mismos adolescentes cuatro años después con el objeto de comprobar la evolución de su trayectoria de integración durante ese intervalo de tiempo. En la tercera y última, se recogió nuevamente después de cuatro años, la información pertinente para comprender hacia donde se habían decantado las trayectorias de integración de los mismos jóvenes anteriormente entrevistados.

Como era de esperar, desde la primera fase de seguimiento se presentaron problemas para la recuperación de la muestra debido entre otras cosas a la gran movilidad de la población inmigrante (cambios de domicilio o localidad, etc.) y a los cambios producidos en las situaciones de los adolescentes al irse haciendo mayores (cambio de colegio, abandono de los estudios, entrada en el mercado laboral, desinterés por el estudio, adquisición de teléfono propio o cambio de número de teléfono, etc.). Con ello se puso a prueba la necesidad de encontrar medios más allá de los conocidos para localizar a los sujetos integrantes de la muestra inicial y es en esa búsqueda que surgió, ya en la primera

\footnotetext{
11 Por "mortandad" de la muestra se entiende la pérdida de casos relativos a la muestra original que habitualmente se va produciendo en las sucesivas oleadas de los estudios longitudinales bien porque los individuos que participaron en la primera oleada resultan ilocalizables o porque no desean continuar participando en el estudio.

12 Aún se están analizando los datos recogidos en la última fase de seguimiento que darán lugar a una publicación a finales del 2020.
} 
fase de seguimiento, la idea de utilizar las redes sociales. Antes de pasar a detallar cómo se utilizaron esas redes, conviene exponer como se desarrollaron la fase inicial y la primera fase de seguimiento. Omitiremos exponer como se desarrolló la última fase ya que no aporta nada nuevo para el objetivo de este artículo que es el de exponer cómo surgió la idea y cómo se utilizaron dichas redes.

\subsection{La encuesta inicial}

En la primera fase del estudio, además de recogerse, como antes se indicó, información sobre la integración de los adolescentes hijos de inmigrantes, se trataba de construir una muestra amplia, representativa y fiable que pudiera ser perseguida en las dos fases siguientes en orden a conocer la evolución habida en los sujetos que compusieran esa muestra. Para ello se tuvo en cuenta que, en España, los niños y niñas de esas edades están escolarizados en su práctica totalidad. De ahí que el primer paso fuera el de obtener una muestra representativa seleccionada aleatoriamente de entre los centros escolares en las ciudades de Barcelona y Madrid y sus respectivas coronas metropolitanas, ciudades que fueron elegidas porque en ellas se hace presente toda la variedad de la inmigración a España. En total, como resultado, participaron en el estudio 180 colegios: 101 en Madrid y 79 en Barcelona. De estos, 111 eran centros públicos y el resto concertados.

Una vez elegidos los centros escolares, el siguiente paso consistió en entrevistar a todos los alumnos de los tres primeros cursos de la enseñanza secundaria obligatoria (ESO) que hubieran nacido en España (segunda generación en sentido estricto) o hubieran sido traídos al país antes de los 12 años (generación 1.5) y que tuvieran al menos uno de los padres nacidos en el extranjero. El trabajo de campo se llevó a cabo principalmente a lo largo del año 2008 aunque comenzó en el último trimestre de 2007. El tamaño total de la muestra fue de 6.905 casos: 3.375 en Madrid y 3.530 en Barcelona. En la siguiente tabla se precisan las principales características de los sujetos de la muestra obtenida en esta primera fase:

Tabla 1. Características Demográficas Básicas de la Muestra Original de la Segunda Generación, 2008.

\begin{tabular}{|c|c|c|c|c|}
\hline Variable & Valores & Barcelona & Madrid & Totales \\
\hline Sexo & $\begin{array}{l}\text { Varón, \% } \\
\text { Mujer, \% }\end{array}$ & $\begin{array}{l}54,13 \\
45,87 \\
\end{array}$ & $\begin{array}{l}48,54 \\
51,46 \\
\end{array}$ & $\begin{array}{l}51,38 \\
48,62 \\
\end{array}$ \\
\hline Edad & $\begin{array}{l}\text { Media } \\
\text { Mediana }\end{array}$ & $\begin{array}{l}13,48 \\
13\end{array}$ & $\begin{array}{l}14,36 \\
14\end{array}$ & $\begin{array}{l}13,91 \\
14\end{array}$ \\
\hline País de Nacimiento & $\begin{array}{l}\text { España, \% } \\
\text { Extranjero, \% }\end{array}$ & $\begin{array}{l}15,94 \\
84,06\end{array}$ & $\begin{array}{l}13,56 \\
86,44\end{array}$ & $\begin{array}{l}14,77 \\
85,23\end{array}$ \\
\hline $\begin{array}{l}\text { Años de residencia en Es- } \\
\text { paña (entrevistados naci- } \\
\text { dos en el extranjero) }\end{array}$ & $\begin{array}{l}\text { Media } \\
\text { Mediana }\end{array}$ & $\begin{array}{l}6,14 \\
5\end{array}$ & $\begin{array}{l}6,78 \\
6\end{array}$ & $\begin{array}{l}6,45 \\
5\end{array}$ \\
\hline Composición Familiar & $\begin{array}{l}\text { Vive con ambos padres } \\
\text { biológicos, } \%\end{array}$ & 65,80 & 66,87 & 66,32 \\
\hline Curso en la ESO & $\begin{array}{l}1^{\circ} \\
2^{o} \\
3^{o}\end{array}$ & $\begin{array}{l}33,59 \\
34,73 \\
31,68\end{array}$ & $\begin{array}{l}8,77 \\
54,40 \\
36,83\end{array}$ & $\begin{array}{l}21,67 \\
44,18 \\
34,15\end{array}$ \\
\hline
\end{tabular}

Fuente: Investigación Longitudinal de la Segunda Generación (ILSEG).

\subsection{La primera encuesta de seguimiento y los problemas de recuperación de la muestra}

Cuatro años después de la primera encuesta a los alumnos, se lanzó la encuesta de seguimiento. Como en otros estudios longitudinales se ampliaba el horizonte de la información buscada hacia variables que recogieran los cambios acontecidos en los jóvenes anteriormente entrevistados al pasar de la adolescencia temprana a la adolescencia tardía y/o primera juventud: prolongación o interrupción de los estudios con entrada al mercado laboral, eventuales cambios en la autoidentificación nacional, etc.

Pero para obtener la información relativa a esta nueva etapa de la integración de los hijos de los inmigrantes, era necesario - dado que se había elegido el método de panel- volver a entrevistar a los mismos sujetos adolescentes que habían sido entrevistados en la fase anterior y para ello era indispensable localizarlos. Así entramos pues en lo central del asunto al que, como se enunció al principio, debían dedicarse estas páginas: ¿cómo localizar a los jóvenes adolescentes que ahora tendrían entre 16 y 18 años y que en muchos casos ya no estarían localizables en los centros de enseñanza donde primero los habíamos encontrado?

Ante todo, por supuesto, buscaríamos localizar a los jóvenes que continuaban matriculados en los mismos colegios en que se les había localizado en la primera fase. Pero este paso rindió poco para reencontrar a los antes entrevistados, 
especialmente en Madrid. De los 3.002 casos conseguidos en la primera fase, por este medio en Madrid solo se pudo recuperar a 793, es decir el $26 \%$ de la muestra original recuperable por poseerse sus señas personales (nombre, dirección, teléfono, etc.). En Barcelona los casos recuperados fueron el 37,8\% (840) de la muestra original, algo más que en Madrid. Evidentemente estas cifras nos dejaban ante una muestra excesivamente reducida y, lo que es peor, claramente sesgada porque sacaban de nuestra consideración precisamente a los muchachos y chicas que hubieran dejado los estudios para buscar un puesto de trabajo o que hubieran avanzado hacia estudios superiores o de otra clase o que no estuviera estudiando ni trabajando. Era necesario, por tanto, buscar nuevos caminos para recuperar más casos de la muestra inicial a fin de que el estudio tuviera sentido.

Con una baza podíamos contar, aunque después resultó también de alcances limitados: en la encuesta inicial se había pedido a los entrevistados que comunicaran sus domicilios particulares y números de teléfono -aunque era de temer, como ocurrió, que pasados tres años dejara de ser utilizable buena parte de esta información dada la alta movilidad de la población inmigrada. De hecho, fue solo de 466 y 213 el número de contactos conseguidos por ese camino en Madrid y en Barcelona respectivamente. Y con todo ello apenas se había logrado recuperar el 35,5\% de la muestra original. Era pues necesario encontrar otras vías para continuar con el intento de recuperación de la muestra y ese fue el papel que el equipo de investigación pensó podrían representar las redes sociales de internet.

Los pasos seguidos se resumen en el diagrama de flujos a continuación.

Gráfico 1. Diagrama de Flujos de los Pasos Seguidos en el Rastreo de la Muestra.

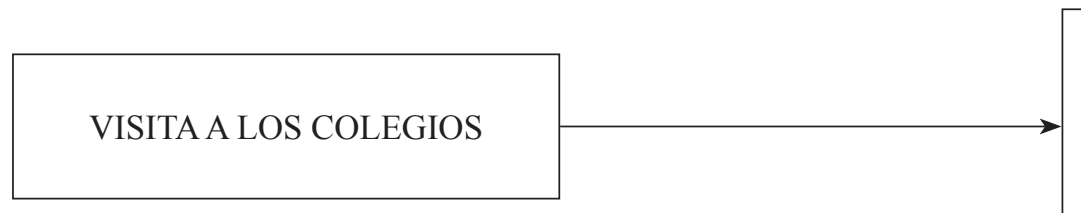

Dificultades:

-Sujeto ha abandonado los estudios

-Sujeto ha cambiado de colegio

-Director del centro se niega a cooperar

Dificultades:

- No tiene teléfono o no responde

- Sujeto se niega a responder

- Sujeto se ha ido de España

LLAMADAS TELEFÓNICAS

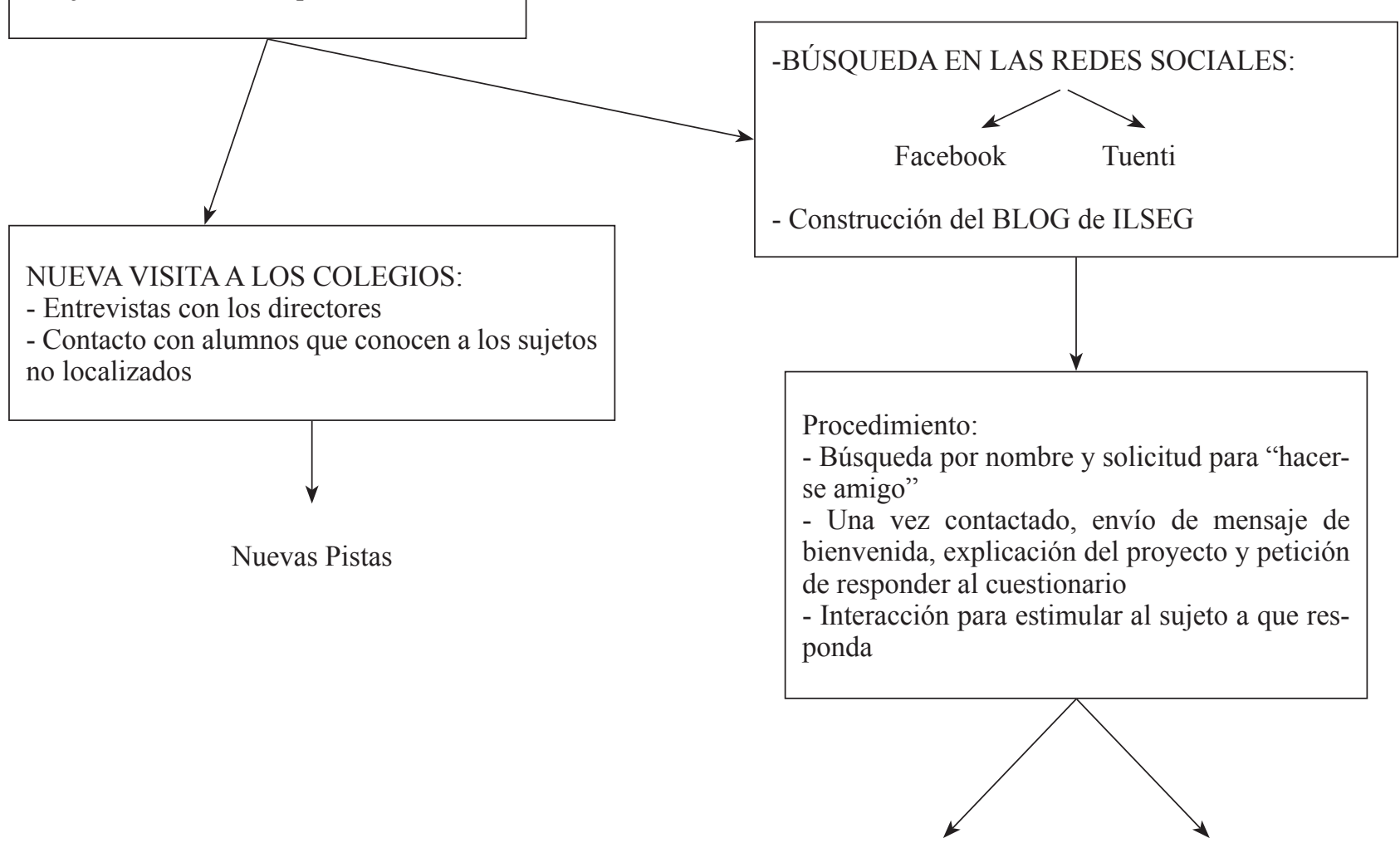

1. Respuesta asistida

2.Teléfono "en línea"

Fuente: Equipo de campo de ILSEG, 2012. 
Como se verá más en detalle después, con el uso adicional de las redes sociales Facebook y Tuenti, se consiguió recuperar el $73 \%$ de la muestra, cifra más que aceptable considerando que había pasado un tiempo relativamente largo desde la encuesta inicial lo que, como se sabe, aumenta las probabilidades de mortandad de la muestra. A continuación detallamos en qué consistió el uso de las redes sociales.

\section{El papel de las redes sociales por internet en la recuperación de la muestra original}

Lo expuesto hasta aquí muestra que inicialmente el estudio había tenido en cuenta alguno de los pasos más comúnmente utilizados en orden a asegurar en la medida de lo posible la recuperación de los individuos de la muestra original en la fase de seguimiento. Es así como ya en la elaboración del cuestionario se había previsto solicitar la dirección de su domicilio y el teléfono a los participantes (en esa etapa no se había contemplado la posible utilización de las redes sociales para localizarlos y por tanto no se les pidió que proporcionaran sus direcciones de correo electrónico ni las direcciones para acceder a su perfil de Facebook o de otras redes sociales como si se hizo en el cuestionario de la segunda fase de seguimiento después de la experiencia de la primera).

De ahí se pasó cuatro años después a hacer el primer seguimiento de los niños y niñas que se habían entrevistado originalmente sin haber mantenido ninguna clase de contacto con ellos, aunque sí con una muestra de sus padres a los que habíamos encuestado en 2010. Pero nada más. Así, después de un primer intento de localizarlos en los mismos colegios de la fase inicial, se pasó a intentar localizar a los no recuperados de esta forma usando la información que ellos mismos nos habían proporcionado en el cuestionario y, todavía, a los que tampoco habíamos podido localizar de esta forma, se intentó rastrearlos mediante el método de contactos colaterales que resultó ser bastante fructífero pero que aún nos dejaba lejos de la meta a alcanzar.

Con esto se nos habían agotado todos los recursos que teníamos a mano para continuar en la búsqueda de los niños y niñas de la muestra inicial que no habíamos conseguido contactar. Cómo se expuso en el primer apartado de este artículo, en los estudios en otros países que han sido analizados por diversos investigadores, lo más común llegados a este paso ha sido el de recurrir a datos administrativos asequibles al público (como por ejemplo en los EE.UU. las direcciones y teléfonos actualizados a través de los números de la seguridad social) o también pagar a empresas que se dedican a rastrear personas y o pueden acceder a datos de distintas fuentes. Eso, sin embargo, en España no es factible como ya señalamos antes. Pero además, el uso de datos obtenidos por algunos de estos procedimientos podría constituir una vulneración de los derechos de la persona, cuestión especialmente delicada tratándose de adolescentes.

En uno de los artículos que hace una revisión de estudios longitudinales en el campo de la salud, sus autores sacan como conclusión que "los estudios longitudinales actuales requieren de aproximaciones creativas para dar cuenta de la complejidad implícita en establecer contacto con las poblaciones inmigrantes". ${ }^{13}$ Tal afirmación es válida no solo para investigaciones con inmigrantes, pero también para las que conciernen a adolescentes y probablemente a otras subpoblaciones que por sus características particulares presentan especiales dificultades de localización. Y tal afirmación describe la situación en la que los investigadores se encontraron en esta investigación al haber agotado todos los recursos disponibles para poder continuar recuperando a los individuos de la muestra inicial. La cuestión es que no valía pensar en cualquier forma de acceder a las personas o a los datos de estas, sino que había que imaginar lugares en los que podían confluir, sino todos, al menos una gran mayoría de los adolescentes. Y fue mediante una sesión de brainstorming entre los miembros del equipo de investigación que surgió la idea de algo que, por otra parte, es bien sabido en general: que en la actualidad ese lugar son las redes sociales. De hecho, prácticamente cualquier estudio actual sobre adolescentes o jóvenes ${ }^{14}$ coincide en el uso de las redes sociales prácticamente por la totalidad de estos y esto era indirectamente corroborado por trabajos de tipo cualitativo con adolescentes hijos de inmigrantes realizados en España por uno de los investigadores principales de ILSEG. Pero además, la mayoría de los estudios coinciden en que el uso de las redes sociales en internet por parte de jóvenes y adolescentes es asiduo lo cual era un indicador de que llegarían a ellos los mensajes que se les cursaran.

Con esta confianza se pensó entonces que, en la práctica, era muy probable que un gran número de los sujetos que se buscaban tuvieran un perfil y estuvieran activos en dichas redes. De todas formas, el equipo investigador buscó también asesorarse con un especialista en redes sociales para saber si estas podían realmente servir al propósito buscado y para que aconsejara sobre qué redes serían las más convenientes para el caso. De ellas entonces, por recomendación suya y por ser la más utilizada por los jóvenes en general se eligió a Facebook y también se tuvo en cuenta a Tuenti, ${ }^{15}$ una red española que era popular en aquel momento entre los jóvenes y sobre todo entre los adolescentes, especialmente en Madrid. Uno de los adolescentes participantes la describió como "una red social hecha para los amigos, más informal y más exclusiva".

Pero ¿por qué podían servir y cómo funcionan estas redes sociales? ${ }^{16}$

\footnotetext{
Sabidó et al. 2014: 81.

González-Anleo (2015).

Tuenti en la actualidad ha dejado de operar como red social.

16 Han colaborado en facilitar la información que aquí se recoge Sileny Cabala, Jaime Goez y Mónica Monguí, miembros del equipo de campo de ILSEG.
} 
Estas dos redes sociales tienen la pretensión de facilitar la comunicación y el intercambio de distintos contenidos de manera virtual entre los "amigos", denominados así los familiares y/o amigos o conocidos que han aceptado una petición de amistad.

Facebook y Tuenti, como muchos saben, sirven para que la persona pueda compartir fotos, entablar una conversación (chat), observar las noticias o estados de otras personas, escribir y recibir mensajes, además de conocer los contactos que tiene un usuario, dando la posibilidad de encontrar amigos en común o contactos de interés, entre otros. A su vez cuenta con un espacio en cada perfil, "muro o wall" en el que los "amigos" pueden escribir o publicar mensajes para que puedan ser vistos por más personas a diferencia de los mensajes privados o "inbox".

Estas dos redes permiten localizar personas poniendo a disposición algunas herramientas que facilitan la búsqueda y contacto.

La búsqueda se puede efectuar a través de:

- Correo electrónico

- Nombre y apellido

- Nickname (alias) que se pone el usuario para poder ser contactado solo por sus amigos.

Esta búsqueda a su vez puede ser más específica mediante la aplicación de filtros como: "lugar de residencia", "lugar de estudio", "lugar de trabajo", "lugar de origen", entre otros.

Una vez que las personas han sido localizadas, Facebook y Tuenti ponen a disposición dos opciones para poder contactarlas:

- Solicitud de amistad

- Mensaje

No obstante, estas dos opciones de contacto están supeditadas a lo que la persona configura dentro de su cuenta de Facebook y/o Tuenti, ya que cada usuario tiene la posibilidad de autorizar o no la activación de cada una de las herramientas, teniendo como consecuencia que, en ocasiones, se tenga que contactar solo por "mensaje" o solo mediante la "solicitud de amistad". La oportunidad que brindan estas dos redes sociales en la configuración de la privacidad trae como resultado a su vez que en ciertos casos la información y contenidos sean limitados a lo que el usuario quiera compartir (por esta razón hay usuarios a los que no se puede llegar a través de los "amigos" puesto que tienen configurado el no permitir ver a sus contactos).

\section{Cómo se desarrolló en este caso el proceso de búsqueda}

Este trabajo en las redes sociales se inició mediante la apertura en Facebook de dos cuentas para el proyecto, la una para los jóvenes de la muestra de Madrid y la otra para los de la de Barcelona, más otra en Tuenti que prácticamente sólo se utilizó para la muestra de Madrid ya que en el proceso se vio que apenas era utilizada por los adolescentes en Barcelona. En Madrid ocurría lo contrario, que era utilizada más que Facebook.

Una vez abiertas las cuentas (o más bien los perfiles), se procedió a la búsqueda de los jóvenes de la muestra que no se habían localizado por otras vías mediante nombre y apellido, aplicando en ocasiones los filtros de "lugar de residencia", "lugar de origen" o "lugar de estudio". Se envió solicitud de amistad y mensaje privado informando sobre la continuación del proyecto en cuya fase inicial habían participado, invitándolos a que aceptaran a los investigadores como amigos.

A quienes aceptaban la solicitud de amistad se les envió un segundo mensaje privado explicándoles la manera cómo podrían participar en esta segunda fase. En ciertos casos contestaban al mensaje dejando un número de teléfono o solicitando el enlace para responder al cuestionario por internet. En otras ocasiones se logró entablar conversaciones por medio del chat, contestando a través de este medio al cuestionario.

Sin embargo, no todos los mensajes y solicitudes recibieron respuesta, lo cual se intentó superar insistiendo con nuevos mensajes. Además, al observar que existían sujetos que ignoraban los mensajes, se procedió a enviarles una información corta sobre la continuación del proyecto, seguido del enlace del cuestionario, con el objetivo de que pudieran responder a este directamente sin tener que entablar relación con el equipo de campo.

\section{Dificultades y estrategias para resolverlas}

Los medios de búsqueda a los que se ha aludido tropezaron a veces con dificultades no siempre fáciles de superar. A continuación se hace referencia a algunas de las más frecuentes:

- Al principio, debido a la falta de experiencia del equipo investigador, se enviaban de golpe un gran número de mensajes y solicitudes de amistad lo cual llevó a que bloquearan las cuentas por periodos que podían durar de 3 días a 2 meses. Se tuvo entonces que recurrir a abrir otras cuentas o perfiles en las nombradas redes sociales y enviar un número limitado de invitaciones y mensajes por día desde cada una de ellas (se enviaban una media de 10 mensajes y 4 solicitudes de amistad al día desde cada cuenta o perfil). 
- Otra dificultad se derivaba del hecho de que bastantes participantes se identifican en las redes con motes o nicknames en vez de con su nombre y apellidos (por ejemplo: Marlene Piruletamente feliz, Morenita rika, Caballero negro). Poco podía hacerse entonces para llegar a identificarlos con su identidad real que es la que constaba en los archivos excepto confiar en que otros de los adolescentes contactados los conocerían y pudieran ayudar en su identificación.

- Una tercera dificultad se derivaba del hecho de que es frecuente entre los jóvenes y adolescentes el encontrarse con demandas de información más o menos personal de tipo comercial o incluso con propuestas peligrosas, escudándose en proyectos de promoción juvenil. Esto a no pocos les hacía desconfiar. Para contrarrestar desconfianzas, se pidió a los que ya habían contestado por otras vías al cuestionario que se unieran como amigos a los perfiles de la investigación en las redes sociales. De esta manera, los contactados por Facebook o Tuenti podían encontrar en dichos perfiles a sujetos conocidos. Asimismo, con un cambio de orientación para conseguir los mismos fines, se optó por publicar en la red un blog resumen del estudio -del que luego se hablará más- que mostraba con claridad cuál era su contenido e invitaba a colaborar con el mismo.

- Hubo también unos pocos participantes que abandonaron porque al abrir la aplicación no supieron rellenar el cuestionario on-line. Se trataba de los menos expertos en el uso de nuevas tecnologías. A muchos de estos participantes, se les tuvo que ir asistiendo mediante el chat, pudiéndose alargar una entrevista de 15 a 20 minutos en una o dos horas.

- Si bien el uso de Facebook y Tuenti permitió encontrar casos ilocalizables por otros medios, no significó que todos los adolescentes localizados respondieran al cuestionario. Es más, el que aceptaran las invitaciones de amistad de los investigadores no significaba que permitieran continuar el contacto con ellos. E incluso cuando sí permitían continuar ese contacto con ellos, podía ocurrir que hubiera que invertir mucho tiempo insistiendo para que al fin respondieran al cuestionario.

\section{Movilización e incentivos}

La interacción con los contactados a través de las redes no se redujo a solo pedirles que completaran sus cuestionarios. Además, se les invitaba a "unirse" al equipo de campo y así formar parte del estudio. Este proceso de formación de redes tomó varias formas: primero, enviando regularmente mensajes informando a los entrevistados de los progresos hechos y dando la bienvenida a nuevos participantes; segundo, creando un Blog ${ }^{17}$ en el cual se invitaba a los entrevistados a colgar videos, fotografías y mensajes sobre sí mismos y sobre sus proyectos; tercero, solicitando pistas para localizar a otros compañeros de colegio que habían participado en la encuesta original o pidiéndoles que los localizaran y les animaran a participar en la encuesta.

Cuadro 1. Portada del Blog dirigido a los adolescentes participantes del estudio ILSEG.

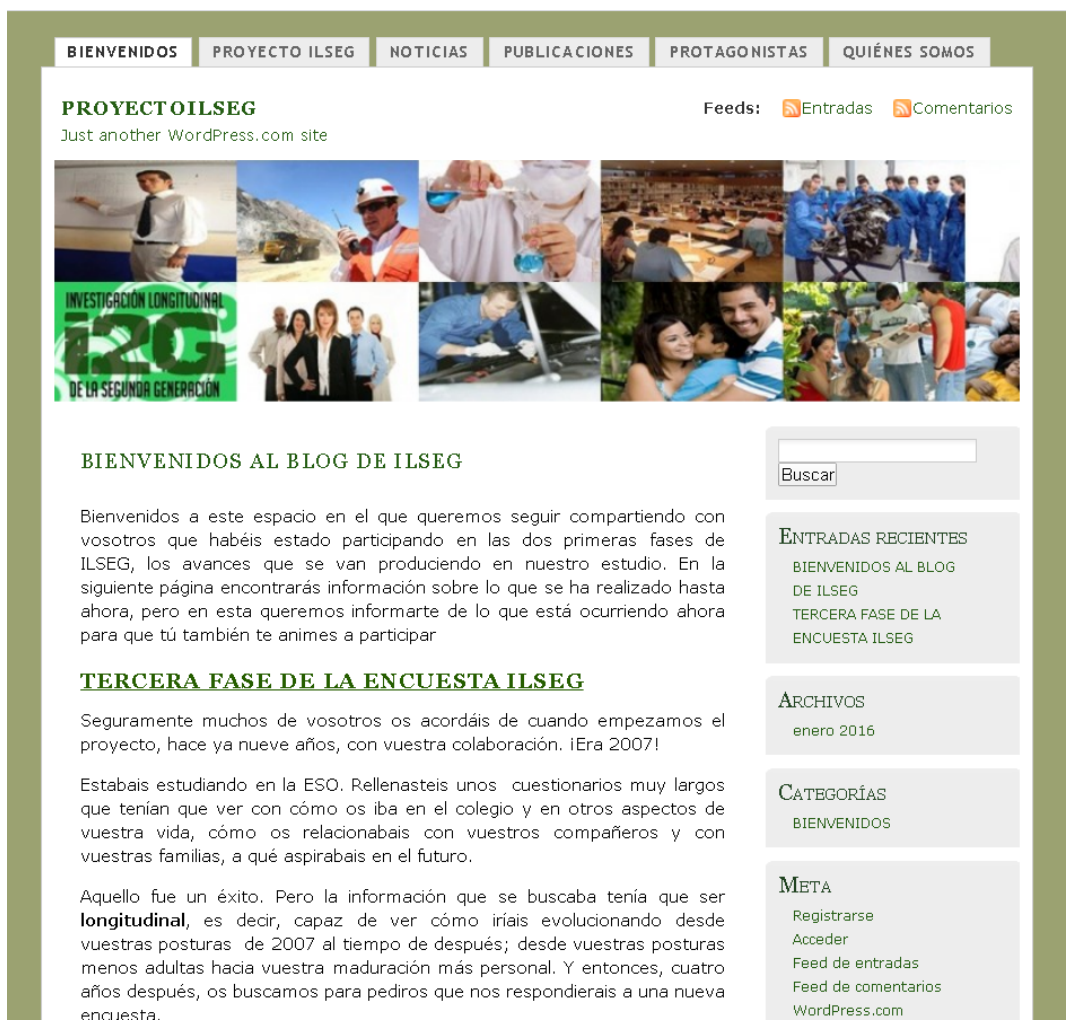

El Blog está accesible en el siguiente enlace: https://proyectoilseg.wordpress.com/bienvenidos/ 
Para estimular las derivaciones, se propició un proceso de emulación entre los graduados de los colegios incluidos en la primera encuesta del estudio. Se incluyó una lista de los centros en el Blog y se invitaba a los entrevistados a apoyar al equipo del proyecto proporcionando pistas de manera que su colegio se convirtiera en uno de los "ganadores" por haber completado la lista de participantes originales. En el mismo mensaje se les estimulaba a colgar en el Blog sus videos, fotos y otros materiales que quisieran. Y para que no faltara algún apoyo material a las demandas que se les hacían, el estudio organizó la rifa de 3 premios de 1500 euros cada uno a sortear entre todos los que hubieran contestado al cuestionario por cualquiera de los medios gracias a los cuales se había localizado a los sujetos.

\section{Resultados}

El equipo investigador no tenía noticia de que anteriormente se hubiera llevado a cabo una exploración parecida de las posibilidades que ofrecen las redes sociales virtuales para localizar a los sujetos en estudios longitudinales con adolescentes. Pero los resultados de este estudio mostraron que esta vía es posible y muy fructífera. Sin la exploración mediante las redes sociales, la proporción de los localizados hubiera sido solamente el $42 \%$ de la muestra original. Es decir, una proporción que no hubiera sido suficiente para legitimar las conclusiones del estudio y que hubiera limitado mucho los análisis que se hubieran podido realizar con los datos. En cambio, la cifra de los sujetos recuperados (73\%) sí que alcanzó un porcentaje -incluso lo superó- que se da por válido entre los estudiosos para este tipo de investigaciones con adolescentes cuando ha habido un lapso de tiempo tan extenso entre una y otra oleada.

Conseguir que la recuperación de la muestra mediante el recurso a las redes sociales en internet contribuyera de manera tan importante al resultado final no fue empresa fácil. Puede ser ilustrativo de esto la proporción de respuestas finalmente obtenidas por relación al número de mensajes iniciales enviados, sin contar los múltiples mensajes insistiendo, que fue del $47 \%$.

La siguiente tabla presenta la muestra final de la encuesta de seguimiento de ILSEG desglosada según el modo de recuperación.

Tabla 2. Número y Proporción de Entrevistas Obtenidas a Través de los Distintos Medios en la Encuesta de Seguimiento.

\begin{tabular}{|l|c|c|c|c|c|}
\hline Ciudad & $\begin{array}{c}\text { Entrevistados } \\
\text { en los colegios }\end{array}$ & $\begin{array}{c}\text { Entrevista } \\
\text { telefónica }\end{array}$ & $\begin{array}{c}\text { Pistas del Personal (profeso- } \\
\text { res y compañeros de clase) } \\
\text { de los Colegios }\end{array}$ & $\begin{array}{c}\text { Redes Sociales en } \\
\text { Internet }\end{array}$ & TOTAL \\
\hline Madrid & 342 & 466 & 217 & 886 & 1911 \\
\hline \multirow{2}{*}{ Barcelona } & 17,9 & 24,4 & 11,3 & 46,4 & 100 \\
\hline \multirow{2}{*}{ Total } & 840 & 203 & 125 & 732 & 1900 \\
& 44,2 & 10,7 & 6,6 & 1618 & 3811 \\
\hline
\end{tabular}

Fuente: Investigación Longitudinal de la Segunda Generación (ILSEG).

En ella queda reflejada la importante contribución de las redes sociales en internet a la recuperación de la muestra original, lo cual resulta aún más impactante visto en el gráfico que sigue.

Gráfico 2. Proporción de entrevistas obtenidas a través de los diferentes medios en la encuesta de seguimiento.

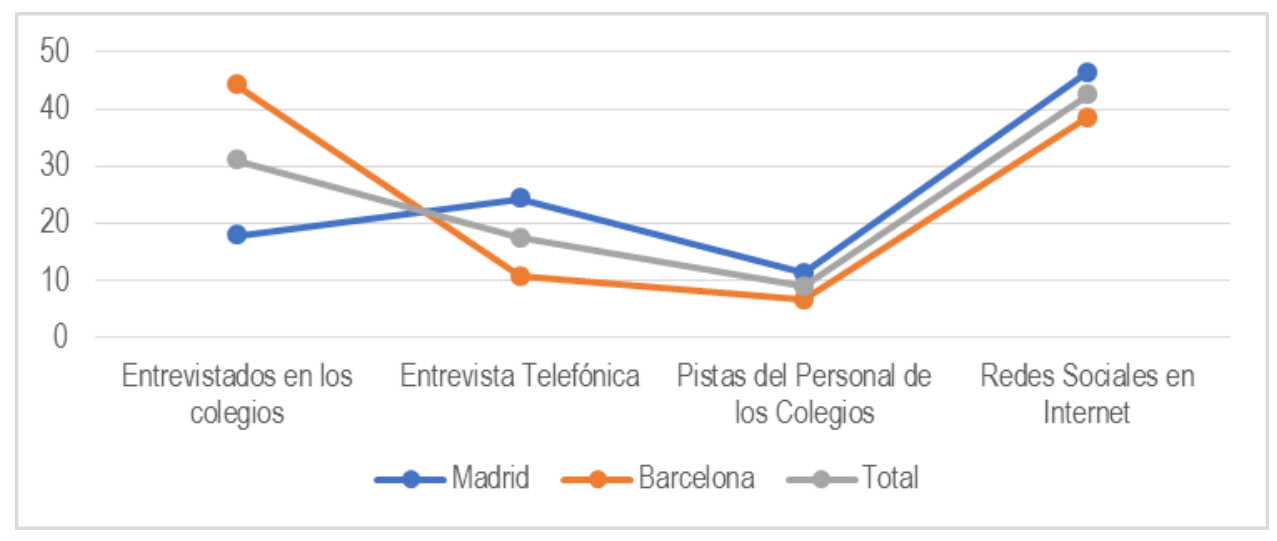

Fuente: Investigación Longitudinal de la Segunda Generación (ILSEG). 
Además de incrementar espectacularmente la cifra de los encuestados localizados, el esfuerzo de localización a través de las redes sociales tuvo otras consecuencias importantes. De una parte, se convirtió por sí misma en una fuente de información acerca de las actividades, opiniones y planes de un número considerable de estos jóvenes abriendo con ello la posibilidad de nuevos estudios. De otra parte, creó un fenómeno social emergente en forma de una red de contactos de crecimiento rápido entre los miembros del equipo del proyecto y los encuestados, así como entre estos últimos.

\section{Conclusiones}

Esta investigación ha mostrado como las redes sociales pueden ser un instrumento muy fecundo en la recuperación de muestras en estudios longitudinales de panel cuando los sujetos son adolescentes o jóvenes. Ahora bien, esa utilidad se ha mostrado al haber sido utilizada en combinación con el uso de otros medios. Los obstáculos que hemos visto existen en las redes sociales para acceder a los usuarios pueden imponer serias limitaciones para recuperar por sí sola la totalidad de la muestra recuperable.

Es posible que muchos de los individuos recuperados previamente a través de otros medios convencionales, no hubieran sido localizables por vía de las redes sociales al utilizar nombres ficticios en estas o tener bloqueado el acceso a sus perfiles por personas ajenas a su círculo de amigos y conocidos. Asimismo, el uso de estos medios con el propósito de recuperar la muestra mostró ser un trabajo laborioso no solo de búsqueda sino también de motivación e insistencia continuada, todo ello requiriendo una considerable inversión en tiempo que pudiera no ser viable en todas las investigaciones.

Por otra parte, no sabemos qué procesos de autoselección pudieran estar operando entre los adolescentes según las distintas maneras de presentarse y dar o no acceso a sus perfiles los cuales, de producirse de forma sistemática y corresponder con uno o varios subgrupos de la población objeto del estudio, podrían introducir sesgos considerables en las muestras recuperadas. Hay que señalar sin embargo que ello no ocurrió en el estudio ILSEG como demostraron las pruebas que se hicieron con el total de la muestra recuperada para ver hasta qué punto se correspondía con la muestra inicial. Pero es una posibilidad que no se debe descartar de antemano.

Todo lo anterior no obsta para concluir que el uso de las redes sociales virtuales puede constituir un recurso muy importante en la recuperación de muestras tratándose de adolescentes. De hecho, en este caso este medio superó a los demás en cuanto a localización y en cuanto a respuesta por parte de los adolescentes de la muestra inicial. Sería un recurso para utilizar en combinación con otros y no por sí solo. Pero, por último, debemos también valorar de este instrumento las perspectivas de conocimiento que su uso abre a los investigadores más allá de la recuperación de la muestra buscada al darles acceso a sus perfiles, lugar en el que los adolescentes tienden a colgar mucho de lo que les interesa e importa en sus vidas.

\section{Referencias bibliográficas}

Aparicio, R., Portes, A. (2014). Crecer en España. La integración de los hijos de inmigrantes. Barcelona: Obra Social la Caixa.

Boys, A. et al. (2003). Minimizing respondent attrition in longitudinal research: Practical implications from a cohort study of adolescent drinking. Journal of Adolescence, 26, 363-373. https://doi.org/10.1016/S0140-1971(03)00011-3

Bynner, J. (1996). Use of longitudinal data in the study of social exclusion. London: Centre for Longitudinal Studies, UCL Institute of Education.

Delgado, M., Llorca, J. (2004). Estudios longitudinales: concepto y particularidades. Revista Española de Salud Pública, vol. 78, no.2, 141-148. http://scielo.isciii.es/pdf/resp/v78n2/colaboracion1.pdf

Dworsky, A. (2014). Children as Self-Informants in Longitudinal Studies: Substantive Findings and Methodological Issues. En G.B. Melton et al. (ed.), The SAGE Handbook of Child Research (pp. 391-431). London: SAGE Publications. https://doi. org/10.4135/9781446294758

Epstein, J. A., Botvin, G. J. (2000). Methods to decrease attrition in longitudinal studies with adolescents. Psychological Reports, 2000, 87, 139-140.

Firebaugh, G. (2008). Seven Rules for Social Research. Princeton: N.J.: Princeton University Press.

González-Anleo, J.M. (2015). La Generación Selfie. Madrid: Ediciones PPC.

Haller,W., Portes, A., Lynch, S. (2011). Dreams Fulfilled, Dreams Shattered: Determinants of Segmented Assimilation in the Second Generation. Social Forces 89, 733-62. https://doi.org/10.1353/sof.2011.0003

Hobden, K. et al. (2011). Limiting Attrition in Longitudinal Research on Homeless Adolescents: What Works best? Journal of Community Psychology, Vol. 39, No. 4, 443-451. https://doi.org/10.1002/jcop.20445

King, N., Churchill, L. (2000). Ethical Principles Guiding Research on Child and Adolescent Subjects. Journal of Interpersonal Violence, 15(7), 710-724.

Loeber, R., Farrington, D. P.(1994). Problems and solutions in longitudinal and experimental treatment studies of child psychopathology and delinquency. Journal of Consulting \& Clinical Psychology, 62, 887-900. https://doi.org/10.1037/0022-006X.62.5.887

Menard, S. (2007). Introduction: Longitudinal Research: Design and Analysis. En S. Menard (Editor), Handbook of Longitudinal Research: Design, Measurement, and Analysis (pp. 3-12). Nueva York: Elsevier. 
Portes, A., Aparicio, R. (2016). Spanish Legacies. The Coming of Age of the Second Generation. Oakland, California: University of California Press.

Sabidó, H. C. et al. (2014). Una revisión sistemática de estudios longitudinales de cohorte sobre la salud en poblaciones migrantes. Medicina Social (www.medicinasocial.info), volumen 8, número 2, 81-94.

Stephens, R. C. et al. (2002). Research Note: An Empirical Study of Adolescent Student Attrition. Journal of Drug Issues, 22-24, 475-488. https://doi.org/10.1177/002204260703700212

Teague et al. (2018). Retention strategies in longitudinal cohort studies: a systematic review and metaanalysis. BMC Medical Research Methodology 18, 151. https://doi.org/10.1186/s12874-018-0586-7.

Thomas, D. et al. (2010). Cutting the Costs of Attrition: Results from the Indonesia Family Life Survey. Journal of Development Economics. 98 (1), 108-23. https://www.ncbi.nlm.nih.gov/pmc/articles/PMC4332578/ 